Pacific Journal of Mathematics

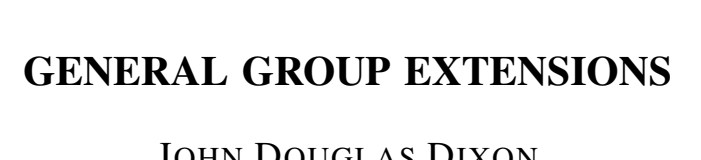




\title{
GENERAL GROUP EXTENSIONS
}

\author{
JOHN D. DIXON
}

Introduction. The aim of this paper is to show how some of the methods useful in studying normal extensions of groups can be used in a problem of more general extensions. The present approach (which might be compared with that of Szep [5]) is made possible because we consider classes of extensions which are still relatively restricted.

If $G$ is an arbitrary subgroup of a group $H$ then the set of all right cosets of $G$ in $H$ forms a mixed group under a naturally defined operation (Loewy [3]). In particular, when $G$ is normal in $H$ then the corresponding mixed group is the ordinary quotient group $H / G$. This paper is concerned with examining properties of the class of those extensions $H$ of a given group $G$ for which the corresponding mixed group is isomorphic to a given mixed group $\Gamma$. As an example of the results, Theorems 2.2 and 2.3 represent analogues of the corresponding theorems of Schreier on factor sets for normal extensions.

The author wishes to record his appreciation to Professor $\mathrm{H}$. Schwerdtfeger for suggesting this problem and encouraging the work.

\section{Mixed groups.}

1.1 Definition. A mixed group is a set $\Gamma$ on which a product $\alpha \beta \in \Gamma$ is defined for certain pairs $\alpha, \beta \in \Gamma$ such that

(i) a nonempty subset $\Delta$ of $\Gamma$ forms a group under the given product and is called the nucleus of $\Gamma$;

(ii) for all $\beta \in \Gamma, \alpha \beta$ is defined if and only if $\alpha \in \Delta$; furthermore, $\alpha \beta=\beta$ if and only if $\alpha=1$, the identity of $\Delta$;

(iii) if $\alpha, \beta \in \Delta$ and $\gamma \in \Gamma$ then $\alpha(\beta \gamma)=(\alpha \beta) \gamma$. (See Loewy [3] and Bruck [2; page 35]. The general properties of mixed groups are derived in Baer [1].)

In particular, if $H$ is a group with a subgroup $G$ then the set of all right cosets of $G$ in $H$ forms a mixed group when the product of two elements is defined by $(G x)(G y)=G x y$ whenever $x \in N(G ; H)$, the normaliser of $G$ in $H$. In this case we denote the mixed group by $H / G$ and note that its nucleus is the quotient group $N(G ; H) / G$ (See Baer [1]).

1.2 Definition. Two mixed groups $\Gamma$ and $\Gamma^{\prime}$ with nuclei $\Delta$ and $\Delta^{\prime}$ respectively are isomorphic under a mapping $\tau$ if $\tau$ is a one-to-one

Received May 23, 1962. 
mapping of $\Gamma$ onto $\Gamma^{\prime}$ such that $(\alpha \tau)(\beta \tau)$ is defined in $\Gamma^{\prime}$ if and only if $\alpha \beta$ is defined in $\Gamma$ and that in that case $(\alpha \beta) \tau=(\alpha \tau)(\beta \tau)$.

Because of part (ii) of Definition 1.1, and isomorphism $\tau$ of $\Gamma$ onto $\Gamma^{\prime}$ induces on the nucleus $\Delta$ of $\Gamma$ a (group) isomorphism onto the nucleus $\Delta^{\prime}$ of $\Gamma^{\prime}$.

As an example, suppose that $H$ is a group with a subgroup $G$ and let $\phi$ be a homomorphism of $H$ onto a group $H^{*}$. If $G^{*}$ is the image of $G$ under $\phi$ and ker $\phi \subseteq G$ then it is easy to show that the mixed group $H / G$ is isomorphic to $H^{*} / G^{*}$. The notation $H / G \geqq H^{*} / G^{*}$ will be used to imply the existence of a homomorphism of $H$ onto $H^{*}$ with this property.

1.3 Definition. If $H$ is a group with subgroup $G$ and $H / G$ is isomorphic to a mixed group $\Gamma$ then $H / G$ is a representation of $\Gamma$ and $H$ is an extension of $G$ by $\Gamma$.

Baer [1; Theorem 3] proves that, except in the case that the mixed group $\Gamma$ is of order 2 and has unit nucleus, every mixed group $\Gamma$ has a representation $H / G$ for some groups $H$ and $G$. (The exceptional case arises because no subgroup $G$ of index 2 in a group $H$ can be its own normaliser.)

1.4. Contrary to the case of normal extensions, not every group $G$ has an extension $H$ by a given mixed group $\Gamma$. From the example of 1.2 , when $H^{*} / G^{*}$ is chosen to be minimal under the quasi-ordering defined there, $G^{*}$ contains no nontrivial normal sabgroup of $H^{*}$. In such a case we call $H^{*} / G^{*}$ a cardinal representation of $\Gamma$. (If $|\Gamma|$ $=n$ is finite, and $H^{*} / G^{*}$ is a cardinal representation of $\Gamma$, then $H^{*}$ is isomorphic to a permutation group of degree $n$ and $G^{*}$ corresponds to a subgroup fixing one letter.) Thus a necessary condition that $G$ should have an extension $H$ by $\Gamma$ is that, for some cardinal representation $H^{*} / G^{*}$ of $\Gamma, G^{*}$ should be a homomorphic image of $G$. Examples show, however, that this condition is not sufficient.

2. Extension functions. As a generalisation of the Schreier factor set used in the theory of normal extensions we consider the extension of a group by a mixed group through the medium of a skew product (cf. Redei [4], Szep [5]).

2.1. Let $G$ be a group and $\Gamma$ be a mixed group with nucleus $\Delta$. We define the skew product $\langle G, \Gamma\rangle$ to be the set $\{\langle a, \alpha\rangle \mid a \in G, \alpha \in \Gamma\}$ on which a binary operation is defined by

$$
\langle a, \alpha\rangle\langle b, \beta\rangle=\langle a f(\alpha, b, \beta), \phi(\alpha, b, \beta)\rangle
$$

with $a, b, f(\alpha, b, \beta) \in G$ and $\alpha, \beta, \phi(\alpha, b, \beta) \in \Gamma$ for some functions $f$ 
and $\phi$ respectively.

We shall denote the identities of $G, \Gamma$ and $\langle G, \Gamma\rangle$ by 1,1 and $\langle 1,1\rangle$ respectively. Furthermore we write $\langle G, \alpha\rangle=\{\langle x, \alpha\rangle \mid x \in G\}$, $\langle a, \Gamma\rangle=\{\langle a, \xi\rangle \mid \xi \in \Gamma\}$ and identify $G$ with $\langle G, 1\rangle$ and $\Gamma$ with $\langle 1, \Gamma\rangle$ under the natural mappings.

2.2 THEOREM. The skew product $H=\langle G, \Gamma\rangle$ is a group with identity $\langle 1,1\rangle$ if and only if the following conditions on $f$ and $\phi$ hold for all $b, c \in G$ and $\alpha, \beta, \gamma \in \Gamma$ :

$$
\begin{array}{cc}
\text { (1) } & f(1, b, \beta)=b \text { and } \phi(1, b, \beta)=\beta ; \\
\text { (2) } & f(\alpha, b, \beta) f(\phi(\alpha, b, \beta), c, \gamma)=f(\alpha, b f(\beta, c, \gamma), \phi(\beta, c, \gamma)) ; \\
\text { (3) } & \phi(\phi(\alpha, b, \beta), c, \gamma)=\phi(\alpha, b f(\beta, c, \gamma), \phi(\beta, c, \gamma)) ; \\
\text { (4) for all } \alpha \in \Gamma \text { there exists } \xi \in \Gamma \text { such that } \phi(\xi, 1, \alpha)=1 .
\end{array}
$$

Proof. (1) is equivalent to $\langle 1,1\rangle\langle b, \beta\rangle=\langle b, \beta\rangle$, that is that $\langle 1,1\rangle$ is a left identity.

(2) and (3) are together equivalent to the associative law

$$
\{\langle a, \alpha\rangle\langle b, \beta\rangle\}\langle c, \gamma\rangle=\langle a, \alpha\rangle\{\langle b, \beta\rangle\langle c, \gamma\rangle\} \text {. }
$$

(4) is equivalent to $\langle 1, \alpha\rangle$ having a left inverse $\langle x, \xi\rangle$ (with $x=$ $\left.f(\xi, 1, \alpha)^{-1}\right)$. However, in that case $\langle a, \alpha\rangle=\langle a, 1\rangle\langle 1, \alpha\rangle$ has a left inverse $\langle x, \xi\rangle\left\langle a^{-1}, 1\right\rangle$ for all $\langle a, \alpha\rangle \in H$.

Thus the stated conditions are necessary and sufficient.

2.3 TheOREM. If the skew product $H=\langle G, \Gamma\rangle$ is a group with identity $\langle 1,1\rangle$ then a necessary and sufficient condition that $\langle G, \Gamma\rangle \mid$ $\langle G, 1\rangle=H / G$ should be isomorphic to $\Gamma$ under the natural mapping

$$
\tau:\langle G, 1\rangle\langle 1, \alpha\rangle=\langle G, \alpha\rangle \rightarrow \alpha \quad(\alpha \in \Gamma)
$$

is that

$$
\begin{gathered}
\phi(\alpha, b, 1)=\alpha \text { for all } b \in G \text { if and only if } \alpha \in \Delta ; \\
\phi(\alpha, 1, \beta)=\alpha \beta \text { when } \alpha \in \Delta .
\end{gathered}
$$

Proof. Since it is clear that $\tau$ is a one-to-one mapping onto $\Gamma$ the theorem follows from Definition 1.2 when we note:

(5) is equivalent to $\langle G, \alpha\rangle \tau \in \Delta$ if and only if $\alpha \in \Delta$;

(6) is equivalent to $\langle G, \alpha\rangle\langle G, \beta\rangle=\langle G, \alpha \beta\rangle$ for $\alpha \in \Delta$.

2.4. A skew product $H=\langle G, \Gamma\rangle$ which satisfies the conditions (1)-(6) will be called an extension of $G$ by $\Gamma$ with functions $f$ and $\phi$. For such an extension it is easily shown that $f$ and $\phi$ have the 
following properties:

(8) for all $\langle a, \alpha\rangle \in H$ there exists a unique $\xi \in \Gamma$ such that $\phi(\xi, a, \alpha)=1$;

(9) the mapping $\gamma \rightarrow \phi(\gamma, a, \alpha)$ permutes the elements of $\Gamma$;

$$
\phi(\alpha, b, \beta)=\alpha \beta \text { when } \alpha \in \Delta \text {. }
$$

2.5 Definition. Two extensions $H_{1}=\langle G, \Gamma\rangle_{1}$ and $H_{2}=\langle G, \Gamma\rangle_{2}$ with functions $f_{1}, \phi_{1}$ and $f_{2}, \phi_{2}$ respectively are called equivalent if there is an isomorphism of $H_{1}$ onto $H_{2}$ which leaves each element of $G$ fixed. If, moreover, the coset $\langle G, \alpha\rangle_{1}$ is mapped onto the coset $\langle G, \alpha\rangle_{2}$ for each $\alpha \in \Gamma$ then $H_{1}$ is said to be biequivalent to $H_{2}$.

Thus if $\theta$ is a one-to-one mapping of $H_{1}$ onto $H_{2}$ then in order for this mapping to be a biequivalence we must have

(i) $\langle a, 1\rangle_{1} \theta=\langle a, 1\rangle_{2}$;

(ii) $\left\{\langle a, \alpha\rangle_{1}\langle b, \beta\rangle_{1}\right\} \theta=\langle a, \alpha\rangle_{1} \theta\langle b, \beta\rangle_{1} \theta$

(iii) $\langle 1, \alpha\rangle_{1} \theta=\left\langle x_{\alpha}, \alpha\right\rangle_{2}$ for some $x_{\alpha} \in G$.

2.6 THEOREM. If $H_{1}=\langle G, \Gamma\rangle_{1}$ and $H_{2}=\langle G, \Gamma\rangle_{2}$ are two extensions of $G$ by $\Gamma$ with functions $f_{1}, \phi_{1}$ and $f_{2}, \phi_{2}$ respectively then $H_{1}$ is biequivalent to $H_{2}$ if and only if there is a function $\alpha \rightarrow x_{\alpha}$ of $\Gamma$ into $G$ such that

$$
\begin{gathered}
x_{\alpha} f_{2}\left(\alpha, b x_{\beta}, \beta\right)=f_{1}(\alpha, b, \beta) x_{\phi_{1}(\alpha, b, \beta)} \\
\phi_{2}\left(\alpha, b x_{\beta}, \beta\right)=\phi_{1}(\alpha, b, \beta) .
\end{gathered}
$$

Proof. If $\theta$ is a biequivalence of $H_{1}$ onto $H_{2}$ then define $x_{\infty}$ by 2.5 (iii). Then $\langle a, \alpha\rangle_{1} \theta=\left\langle a x_{\alpha}, \alpha\right\rangle_{2}$. Therefore

$$
\left\{\langle a, \alpha\rangle_{1}\langle b, \beta\rangle_{1}\right\} \theta=\left\langle a f_{1}(\alpha, b, \beta) x_{\phi_{1}(\alpha, b, \beta)}, \phi_{1}(\alpha, b, \beta)\right\rangle_{2}
$$

and $\langle a, \alpha\rangle_{1} \theta\langle b, \beta\rangle_{1} \theta=\left\langle a x_{\alpha} f_{2}\left(\alpha, b x_{\beta}, \beta\right), \phi_{2}\left(\alpha, b x_{\beta}, \beta\right)\right\rangle_{2}$ and so (11) and (12) are together implied by 2.5 (ii).

Conversely, if we are given (11) and (12) and define $\theta$ as the one-to-one mapping of $H_{1}$ onto $H_{2}$ given by $\langle a, \alpha\rangle_{1} \theta=\left\langle a x_{\alpha}, \alpha\right\rangle_{2}$ then 2.5 (i), (ii) and (iii) follow, so $\theta$ is the required biequivalence.

2.7. Let $H=\langle G, \Gamma\rangle$ be an extension of $G$ by a mixed group $\Gamma$ with functions $f$ and $\phi$. The kernel $\Delta$ of $\Gamma$ is isomorphic to $N(G ; H) / G$ (by 1.1). Therefore $G=\langle G, 1\rangle$ is a normal subgroup (and $H$ is a normal extension of $G$ ) if and only if $\Delta=\Gamma$, and $\Gamma$ is a group. Alternatively, using (5) and (6) we have that $\phi(\alpha, b, 1)=\alpha$ (for all $\alpha \in \Gamma, b \in G)$ as a necessary and sufficient condition that $H$ be a normal extension of $G$.

A second important case is when $H$ is a splitting extension (i.e. 
$\Gamma=\langle 1, \Gamma\rangle$ is a subgroup of $H$ ) so that $H=G \Gamma$ and $G \cap \Gamma=1$. In terms of the extension functions, $H$ is a splitting extension if and only if $f(\alpha, 1, \beta)=1$ for all $\alpha, \beta \in \Gamma$. To prove this we note that, since $\langle 1, \alpha\rangle\langle 1, \beta\rangle=\langle f(\alpha, 1, \beta), \phi(\alpha, 1, \beta)\rangle$, the condition is certainly necessary. It is also sufficient because when it holds we also have

$$
\langle 1, \alpha\rangle^{-1}=\left\langle f(\xi, 1, \alpha)^{-1}, \xi\right\rangle=\langle 1, \xi\rangle \in \Gamma
$$

where $\xi$ is defined as in Theorem 2.2.

2.8 Theorem. An extension $H=\langle G, \Gamma\rangle$ of $G$ by $\Gamma$ with functions $f$ and $\phi$ is a splitting extension if and only if for some function $\alpha \rightarrow x_{\alpha}$ of $\Gamma$ into $G$ we have

$$
f\left(\alpha, x_{\beta}, \beta\right)=x_{\alpha}^{-1} x_{\phi\left(\alpha, x_{\beta}, \beta\right)} .
$$

Proof. Apply Theorem 2.6 with $f_{1}(\alpha, 1, \beta)=1$.

CoROLLARY. If the conditions of the theorem are satisfied then $\Gamma^{*}=\left\{\left\langle x_{\alpha}, \alpha\right\rangle \mid \alpha \in \Gamma\right\}$ is a subgroup of $H$ such that $H=G \Gamma^{*}$ and $G \cap \Gamma^{*}=1$.

\section{REFERENCES}

1. R. Baer, Zur Einordnung der Theorie der Mischgruppen in die Gruppentheorie, Sitz. Heidelberg (1928) Nr. 4.

2. R. Bruck, Survey of Binary Systems, Springer 1958.

3. A. Loewy, Über abstrakt definierte Transmutationssysteme oder Mischgruppon, J. reine angew. Math., 157 (1927), 239-254.

4. L. Redei, Die Anwendung des schiefen Produktes in der Gruppentheorie, J. reine angew. Math., 188 (1950), 201-227.

5. J. Szep, Über eine allgemeine Erweiterung von Gruppen I, II, Publ. Math. Debrecen 6 (1959), 60-71, 254-261.

MCGILL UNIVERSITY AND

California Institute of TeChNology 



\section{PACIFIC JOURNAL OF MATHEMATICS}

\section{EDITORS}

\section{RalPh S. Phillips}

Stanford University

Stanford, California

M. G. Arsove

University of Washington

Seattle 5, Washington
J. Dugundji

University of Southern California Los Angeles 7, California

Lowell J. Paige

University of California

Los Angeles 24, California

\section{ASSOCIATE EDITORS}
E. F. BECKENBACH
D. DERRY
H. L. ROYDEN
E. G. STRAUS
T. M. CHERRY
M. OHTSUKA
E. SPANIER
F. WOLF

\section{SUPPORTING INSTITUTIONS}

\author{
UNIVERSITY OF BRITISH COLUMBIA \\ CALIFORNIA INSTITUTE OF TECHNOLOGY \\ UNIVERSITY OF CALIFORNIA \\ MONTANA STATE UNIVERSITY \\ UNIVERSITY OF NEVADA \\ NEW MEXICO STATE UNIVERSITY \\ OREGON STATE UNIVERSITY \\ UNIVERSITY OF OREGON \\ OSAKA UNIVERSITY \\ UNIVERSITY OF SOUTHERN CALIFORNIA
}

STANFORD UNIVERSITY

UNIVERSITY OF TOKYO

UNIVERSITY OF UTAH

WASHINGTON STATE UNIVERSITY

UNIVERSITY OF WASHINGTON

AMERICAN MATHEMATICAL SOCIETY

CALIFORNIA RESEARCH CORPORATION SPACE TECHNOLOGY LABORATORIES

NAVAL ORDNANCE TEST STATION

Printed in Japan by International Academic Printing Co., Ltd., Tokyo Japan 


\section{Pacific Journal of Mathematics}

\section{Vol. 13, No. 1 \\ March, 1963}

Frantz Woodrow Ashley, Jr., A cone of super-(L) functions............. 1

Earl Robert Berkson, Some metrics on the subspaces of a Banach space....

Felix Earl Browder and Walter Strauss, Scattering for non-linear wave

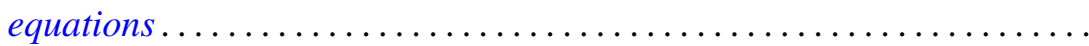

Edmond Darrell Cashwell and C. J. Everett, Formal power series ..........

Frank Sydney Cater, Continuous linear functionals on certain topological

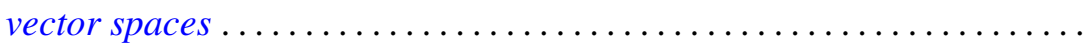

John Douglas Dixon, General group extensions ....................

Robert Pertsch Gilbert, On harmonic functions of four variables with

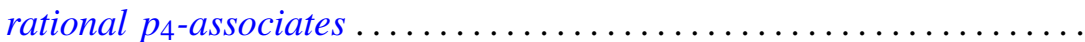

Irving Leonard Glicksberg, On convex hulls of translates ..............

Simon Hellerstein, On a class of meromorphic functions with deficient zeros

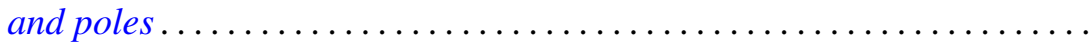

Donald William Kahn, Secondary cohomology operations which extend the

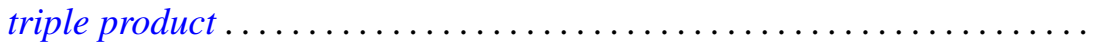

G. K. Leaf, A spectral theory for a class of linear operators .............

R. Sherman Lehman, Algebraic properties of the composition of solutions of partial differential equations ........................... 157

Joseph Lehner, On the generation of discontinuous groups ............. 169

S. P. Lloyd, On certain projections in spaces of continuous functions ...... 171 Fumi-Yuki Maeda, Generalized spectral operators on locally convex spaces ..................................

Donald Vern Meyer, $E^{3}$ modulo a 3-cell

William H. Mills, An application of linear programming to permutation groups.

Richard Scott Pierce, Centers of purity in abelian groups

Christian Pommerenke, On meromorphic starlike functions ...

Zalman Rubinstein, Analytic methods in the study of zeros of

polynomials...

B. N. Sahney, On the Nörlund summability of Fourier series

Tôru Saitô, Regular elements in an ordered semigroup . .

Lee Meyers Sonneborn, Level sets on spheres...........

Charles Andrew Swanson, Asymptotic estimates for limit point

problems .

Lucien Waelbroeck, On the analytic spectrum of Arens . .

Alvin (Murray) White, Singularities of a harmonic function of three

variables given by its series development .............

Kōichi Yamamoto, Decomposition fields of difference sets ...

Chung-Tao Yang, On the action of $\mathrm{SO}(3)$ on a cohomology manifold... 